\title{
A HIGH PRECISION TRIANGULAR LAMINATED ANISOTROPIC CYLINDRICAL SHELL FINITE ELEMENT
}

\author{
H. V. LAKSHMINARAYANA and S. VISWANATH \\ National Aeronautical Laboratory, Bangalore-560017, India
}

(Received 1 February 1976)

\begin{abstract}
The stiffness matrix for a high precision triangular laminated anisotropic cylindrical shell finite element has been formulated and coded into a composite structural analysis program. The versatility of the element's formulation enables its use in the analysis of multilayered composite plate and cylindrical shell type structures taking into account actual lamination parameters. The example applications presented demonstrated that accurate predictions of stresses as well as displacements are obtained with modest number of elements.
\end{abstract}

\section{INTRODUCTION}

Multilayered composite materials are increasingly used in the construction of plate and shell type structures for various industrial and aero-space applications, creating a parallel need for structural analysis procedures which reflect the laminated anisotropic characteristics of such materials. Although the basic equations which govern the elastic behaviour of laminated anisotropic plate and shell structures can be derived[1], analytical solutions of these complex equations, as they pertain to design and analysis, are not available. The analysis of composite structures is an area which calls for an extensive use of digital computers and numerical methods like the finite element method.

The finite element method has proved to be an extremely powerful tool for the solution of problems involving complex geometries, arbitrary loadings and rather general material properties. Considerable advances have been made in the development and application of the finite element method to plate and shell type structures. Much of the work is reviewed by Gallagher[2,3]. According to these reviews, the analysis of thin shells istone of the more difficult problems that has been attempted with the finite element method. The requirements upon valid minimum potential energy solutions in thin shell finite element analysis are extremely difficult to satisfy, and the satisfactory formulations are therefore relatively complicated. Further, for tackling problems involving description of arbitrary boundaries triangular and quadrilateral elements are obviously best suited, The most reliable and sophisticated triangular shell element formulations are those due to Dupuis [4], Cowper et al.[5], Argyris and Scharph [6] and Dawe [7]. The formulation due to Cowper et al. [5] has also been converted into a triangular cylindrical shell finite element by Lindberg and Olson [8]. The salient features of this element are: (i) The element is fully conforming, of the displacement type and of arbitrary triangular shape, (ii) Uses higher order interpolation polynomials and has 36 degrees of freedom, (iii) Transverse displacement function contains a complete quartic polynomial plus some higher degree terms and allows cubic variation of normal slope along each edge. (iv) Tangential displacement functions are complete cubic polynomials to yield a quadratic variation of stresses within the element, (v) Gives accurate predictions of displacements as well as stresses, (vi) Satisfies sufficient conditions to guarantee rapid convergence, (vii) Permits reliable and direct evaluation of stresses at nodes, (viii) The element stiffness matrix is formulated in exact closed form, (ix) Results in a smaller overall problem size and (x) The versatility of the formulation enables its use in the analysis of flat plates in either membrane or flexural behaviour and for the coupled membrane-flexural behaviour of cylindrical shells. The formulations of this element as given in Ref. [8] is confined to homogeneous isotropic materials. The present work is concerned with the generalization of this formulation to take into account the laminated anisotropic characteristics of multilayered composite materials.

The purpose of this paper is to present the formulation and some useful applications of a high precision triangular laminated anisotropic cylindrical shell finite element. In order to perform composite structural analysis, a computer program incorporating this element has been developed. Distinct membrane and flexural stiffnesses are retained permitting analysis of composite structures subjected to inplane and bending loads. The applications presented encompassing fiat plates in plane stress and in flexure and circular cylindrical shells clearly indicate the usefulness of the element in composite structural analysis.

\section{FORMULATION}

The geometry of an arbitrary triangular cylindrical shell element is shown in Fig. 1. The global cylindrical coordinate system is $X, Y$ and $\boldsymbol{\xi}, \eta$ are taken as local coordinates for the element. $R$ is the radius of curvature of the reference surface of the shell and $t$ is its wall thickness. The dimesnions $a, b, c$ of the triangle $1,2,3$ and the rotation angle 6 are easily derived in terms of the global coordinates of the vertices [9].

The strain energy of a thin cylindrical shell in global coordinates is given by

$$
U=\frac{1}{2} \iint\left\{[N]^{T}\left[\varepsilon^{0}\right]+[M]^{T}[x]\right\} d x d y
$$

where

$[N]^{T}=\left(N_{X}, N_{Y}, N X Y\right)$ are the membrane forces

$[M]^{\mathrm{T}}=\left(M_{X}, M_{Y}, M X Y\right)$ are the bending stress resultants

$[\boldsymbol{\epsilon}]^{T}=\left(\epsilon_{X}{ }^{0}, \epsilon_{Y}{ }^{0}, \gamma_{X Y}{ }^{0}\right)$ are the reference surface strains and $[\chi]^{T}=\left(\chi_{X}, X_{Y}, \chi_{X Y}\right)$ are the bending curvatures.

For an arbitrarily laminated anisotropic shell, the con- 


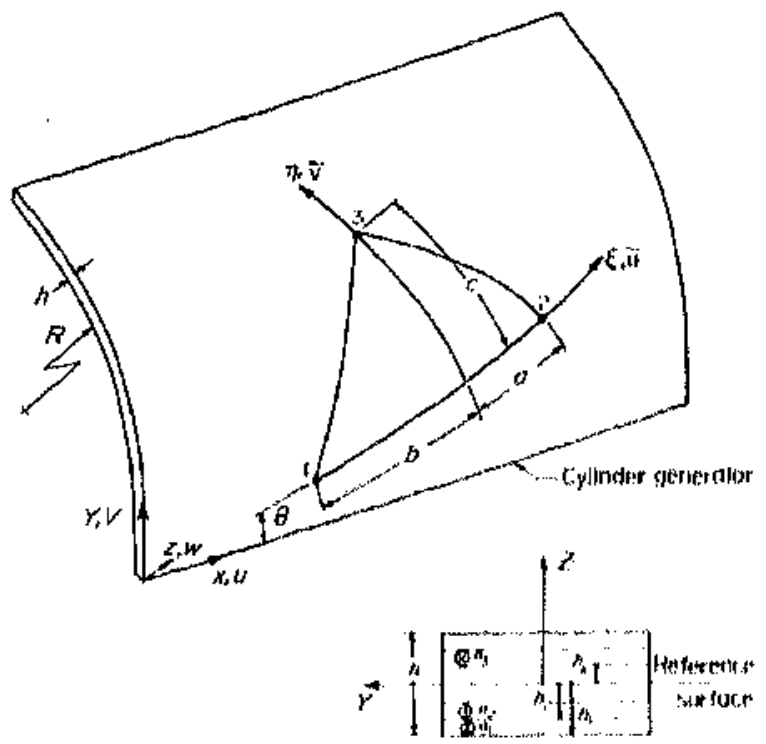

Wall detulls

Fig. 1. Cylindrical shell triangular finite ecement Geometry and coordinate systems,

stitutive relations are expressible in the form 11$\}$

$$
\left[\begin{array}{l}
N \\
M
\end{array}\right] \therefore\left[\begin{array}{ll}
A & B \\
B & D
\end{array}\right]\left[\begin{array}{l}
E^{\prime \prime} \\
X
\end{array}\right]
$$

where $[A],[B],[D]$ are the membrane, interaction and bending stiff ness matrices of the laminate.

Substituting eqns (2) in (1), we get

$$
\begin{aligned}
& U=\frac{1}{2} \iint\left[\left(\epsilon^{*}\right]^{r} \mid A\right]\left[\left.\epsilon^{n}|+| x\right|^{r} \mid B\right] t e^{\prime \prime} \mid \\
& +\left[\left.\epsilon^{\prime \prime}\right|^{r}[B][x]+\mid x\right]^{J}[D|| x \mid) \mathrm{d} x \mathrm{~d} y .
\end{aligned}
$$

Using Novozhilov's thin shell theory, the strain-displacement relations are [10]

$$
\begin{aligned}
& {\left[\epsilon^{(1)}\right]\left[\begin{array}{l}
U_{X} \\
V_{y}|W| R \\
U_{Y}+V_{X}
\end{array}\right]}
\end{aligned}
$$

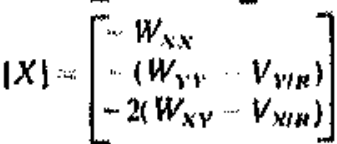

where $U, V, W$ are the displacement components in the global coordinate system $(X, Y, Z), W$ being positive when radially outward. Note that subscript! and (5) denote differentiation.

Substituting for $\left[c^{\text {" }}\right]$ and $|x|$ from eqns (4) possible to express the strain energy given I matrix form as

$$
\text { U } \frac{1}{2} \iint\left\{(d)^{2}[L][d]\right\} \mathrm{d} x \mathrm{~d} y
$$

lequns (4) Id (5), itis aqu (3) i

where

$$
[d]^{I} \quad\left(U_{X}, U_{Y}, V_{X}, V_{Y,} W, W_{X X}, W_{X Y} V_{Y Y}\right)
$$

and the strain encrgy matrix $[I$,$] is given in ble 1$.

If $\ddot{U} . V$ denote reference surface displace ints in the local coordinate system $(\xi, \eta)$, the transfor itionfrom local to global coordinates is

$$
[d]:\left|R_{4}\right|[\tilde{d} \mid
$$

where

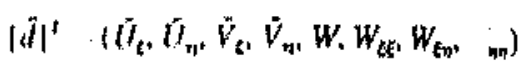

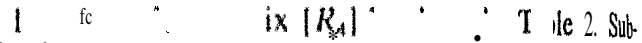
stituting eqn (7) In eqn (6), the strain et 'gy of the element is given by

$$
\text { U. } \left.\left.\frac{1}{2} \iint\{d]^{s}[\pi] \| d\right]\right\} d \xi d \eta
$$

(19)

where

$$
[i]\left|R_{d}\right|^{*}[L]\left[R_{4}\right]
$$

with the area integration to be carried ove he triangle 123.

The assumed displacement functions for te element are|5]

$$
\begin{aligned}
& \text { di. } \sum_{i=1}^{\mid l i} a_{1} \xi^{n+1} \eta^{n_{3}}
\end{aligned}
$$

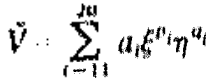

\begin{tabular}{|c|c|c|c|c|c|c|c|}
\hline$A_{11}$ & $\begin{array}{l}A_{11} \\
A_{11}\end{array}$ & $\begin{array}{l}A_{11}+\frac{2 B_{1}}{R} \\
A_{11}+\frac{2 B_{n}}{K} \\
A_{n}+\frac{4 B_{n}}{R}+\frac{4 D_{n}}{R^{2}}\end{array}$ & 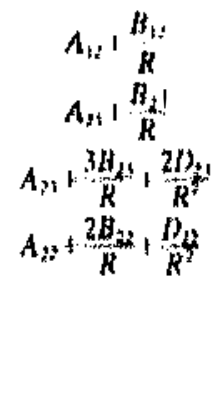 & 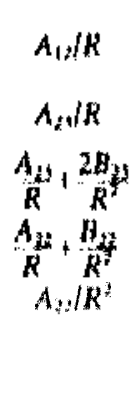 & 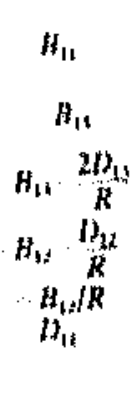 & $\begin{array}{c}2 B_{11} \\
2 B_{41} \\
2 H_{14} \cdots \frac{4 D_{32}}{R} \\
2 H_{31} \cdot \frac{2 D_{3}}{R} \\
2 B_{31} / R \\
2 D_{11} \\
4 D_{31}\end{array}$ & 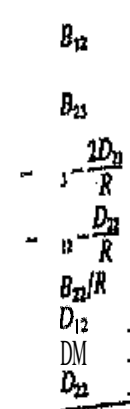 \\
\hline
\end{tabular}

$$
\begin{aligned}
& W \ldots, \mathbf{F}_{n} a_{i}^{k^{\prime}} i \eta^{*}
\end{aligned}
$$

where $m_{i}, n_{i}, n_{i}, r_{i}$ and $s_{i}$ arc just integers Is $^{2}$

The element stiffness matrix is obtained rom a calculation of its strain energy. The displacem t functions of eqns (9) are substituted into cqn (8) and $\mathbf{t l}$ integration curried oul to yield

$$
\text { II. } \left.\sum_{2}^{1}[\alpha]^{\prime} \mid k\right]|\alpha|
$$

$(10)$

Table I. Strain energy matrix |l.1 
Table 2. Rotation matrix $\left[\boldsymbol{R}_{4}\right]$

$\left[\begin{array}{cccccccc}\hline C C & -S C & -S C & S S & 0 & 0 & 0 & 0 \\ S C & C C & -S S & -S C & 0 & 0 & 0 & 0 \\ S C & -S S & C C & -S C & 0 & 0 & 0 & 0 \\ S S & S C & S C & C C & 0 & 0 & 0 & 0 \\ 0 & 0 & 0 & 0 & 1 & 0 & 0 & 0 \\ & & & & & C C & -2 S C & S S \\ & & & & & \text { SC } & C C-S S & -S C \\ & & & & & S S & 2 S C & C C\end{array}\right]$

where

$$
\begin{aligned}
& \mathrm{CC}=\cos ^{2} \theta \quad S S-\sin ^{2} 6 \\
& S C=\sin \theta \cos \theta
\end{aligned}
$$

where $[a]$ is a 40 -column vector of the polynomial coefficients $\boldsymbol{a}_{\boldsymbol{i}}$. The entries of the stiffness matrix $[k]$ are determined in closed form and are given in the Appendix.

The generalized displacements in global coordinates chosen for the element are

$$
\begin{aligned}
{[W]^{\mathrm{T}}=} & \left(U_{1}, U_{X 1}, U_{Y 1}, V_{1}, V_{X 1}, V_{Y 1}, W_{1}, W_{X 1}, W_{Y 1}\right. \\
& \left.W_{X X 1}, W_{X Y 1}, W_{Y Y 1}, U_{2} \ldots, U_{31}, \ldots, W_{Y Y 3}\right)
\end{aligned}
$$

Further steps to be followed in the deviation of the $36 \times 36$ stiffness matrix relating the generalized displacements [ $W]$ and the corresponding generalized forces, and consistent load vector corresponding to uniform pressure load are very similar to those for the shallow shell element [5].

\section{COMPUTER PROGRAM}

The stiffness matrix and consistent load vector for the high precision triangular laminated anisotropic cylindrical shell finite element has been coded into a composite structural analysis program. Distinct membrane and flexural stiffnesses are retained permitting analysis of composite structures subjected to inplane and bending loads. The resulting force-displacement equations are solved using Choleski square root decomposition method for fixed bandwidth problems. Stress resultants and individual layer stresses are directly calculated at the nodes using the generalized displacements, constitutive relations and appropriate transformation equations. With basic lamina elastic constants and laminate details as input, the program evaluates the laminate stiffnesses for each element. It is therefore possible to analyse laminates of varying thickness taking into account the actual lamination parameters. The computer output consists of generalized displacements, stress resultants, membrane and bending strains, individual layer stresses with reference to both laminate and lamina reference axes at each node. With this information it is possible to make strength prediction of practical composite structures using appropriate failure criteria [1].

\section{APPLICATIONS}

The versatility of the element formulation enables its use in the analysis of laminated anisotropic flat plates in either membrane or flexural behaviour and for coupled membrane-bending behaviour of circular cylindrical shells. The following example applications illustrate the usefulness of the element in composite structural analysis. All calculations are carried out on an IBM 370/155 machine using double precision arithmetic.
Boron-epoxy composite laminate with a circular hole under axial tension

In Fig. 2 is shown a boron-epoxy composite panel consisting of nine plies of $[0 / \pm 45 / 0 / 90]_{s}$ laid up with a central circular hole under uniaxial tension. Symmetry of the loading, geometry and material properties make the analysis of only one quarter of the panel sufficient. The finite element idealization of the quarter panel illustrated in Fig. 3 uses 51 elements with 37 nodes resulting in 186 degrees of freedom. Typical stress distributions calculated at the nodes are shown in Figs. 4 and 5 along with the results of anisotropic elasticity solution to the problem of an infinite plate with a circular opening [11]. The present results appear to have converged quite rapidly. The predicted tensile and compressive stress and strain concentration factors given in Table 3 together with results of anisotropic elasticity solution for the plate clearly demonstrate that the element gives excellent accuracy.

An identical problem has been analysed in Ref. [12] using linear-strain triangular elements (twelve degrees of freedom). One hundred and forty one elements with 320 nodes (598 degrees of freedom) were used to describe

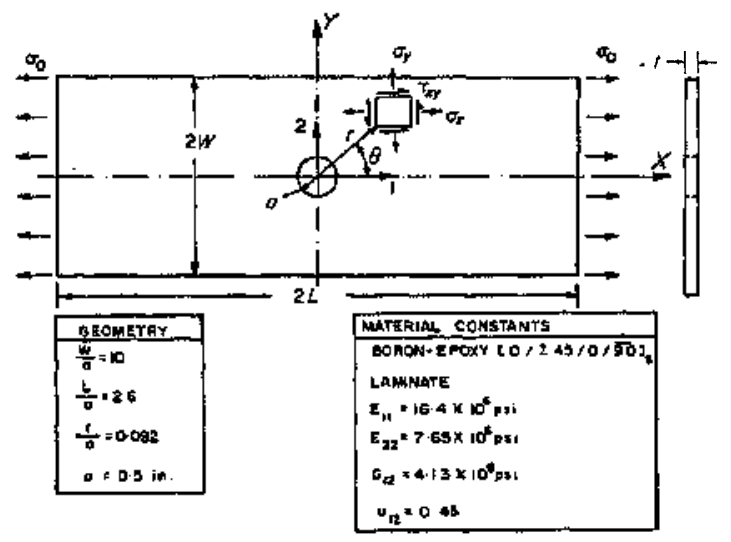

Fig. 2. Boron-epoxy laminate with a circular hole under axial tension.

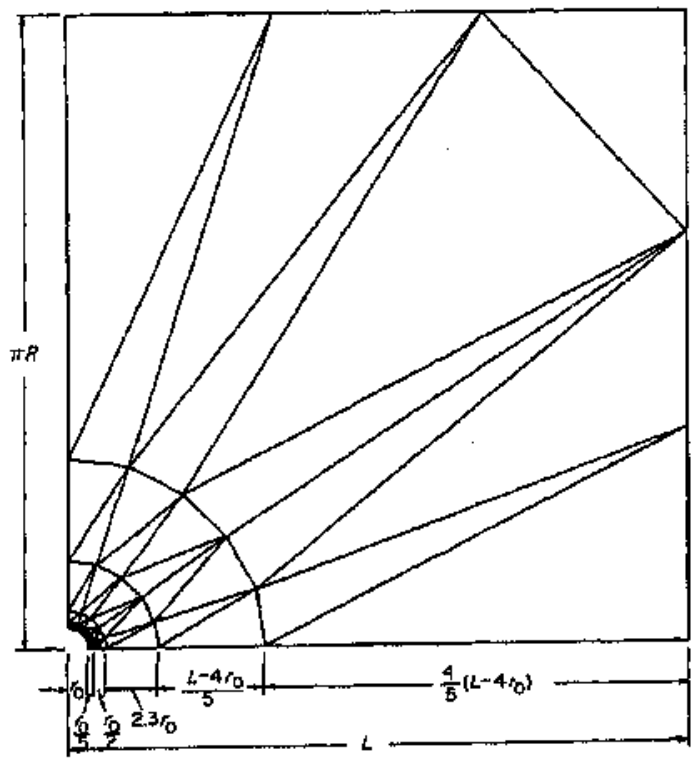

Fig. 3. 5 x 5 Finate element grid. 


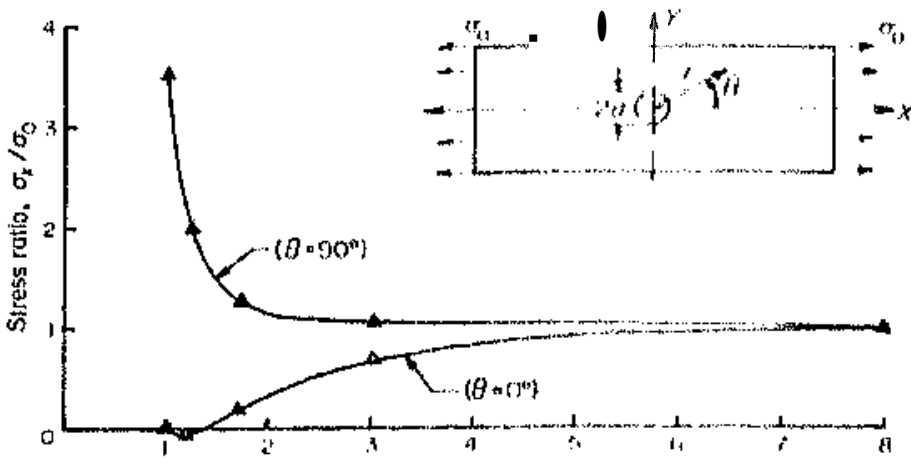

Fig. 4, Axial stress distribution along $X$ and $Y$ axcs.

Analytical solunion: A. finite element resul

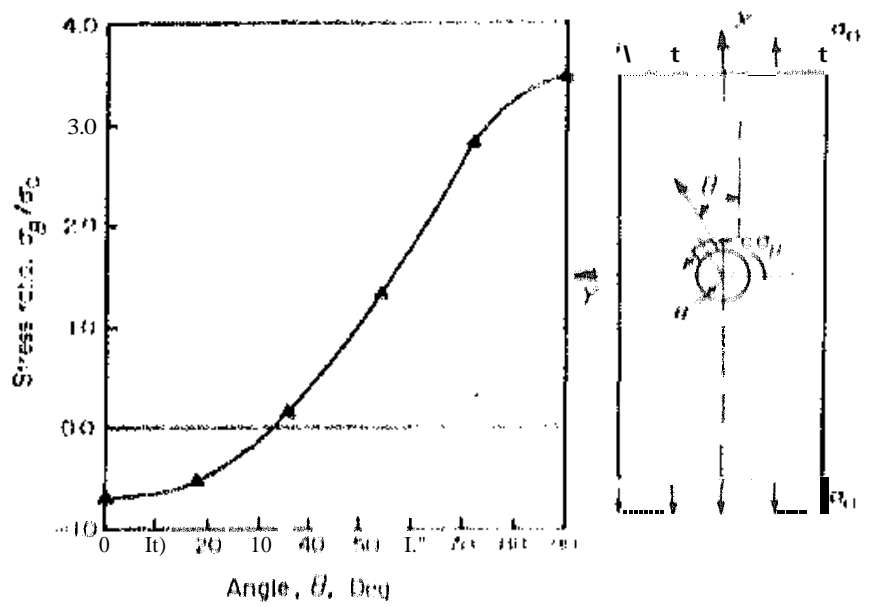

Fig. 5. T'angential stress distribution along hole boundary. , Anatylinal sulution: $A$, finite element re ts.

the quarter panel. The predictions are also given in Table 3 for comparison. The fact that the present formulation yields better accuracy with about one-third the problem size demonstrate the superiority of the element.

The problem has also been analysed experimentally using straingages, photoclastic coatings and moire lech. niques in Ref. (12J. Average predictions are given in Table 3. The close agreement between the present results and experimental data readily verifies the adequacy of the assumed laminated anisotropic model in the unalysis as u first approximation to the actual multilayered com. posite material behaviour.

Clamped glass -epoxy composite square plate subjected to uniform pressure load. Figure 6 shows a glassecpoxy composite square plate with clamped edges subjected to uniform pressure load. Symmetry allows the analysis to be limited to one quarter of the plate. Arrangement of the elements in a quadrant of the plate are Fig. b. Predicted deflection distributions lines of the plate are shown in Fig. 7 al results from an approximate analytical sol Ref, [13]. This inalytical solution assu deflection profiles along the centrelines Whereas, as it should be, the present ana different deflection profiles parallel and pes the tibre direction. This prediction is $\mathrm{i}$ agreement with experimental sbservations tional composite plates in flexure using techniques [ [4]. Bending moment distributi centrelines of the plate shown in Fig. $\mathrm{H}$ are and can be convidered to have converged eal results given in Table 4 can be con accurafe than the solution available in[13]. astexsment of actual accuracy of the pred io shounin ong cats Ig wilh tix on givet it s identivel the plate sis prediless indicular th qualitaline in unidtirs holograptic 5 allong the irly smooth well. Typis lered noce owevts, an cod stressto

Table 3, Boronepoxy laminate with a circtilnr hole undet uxial tenvion compurivon of results

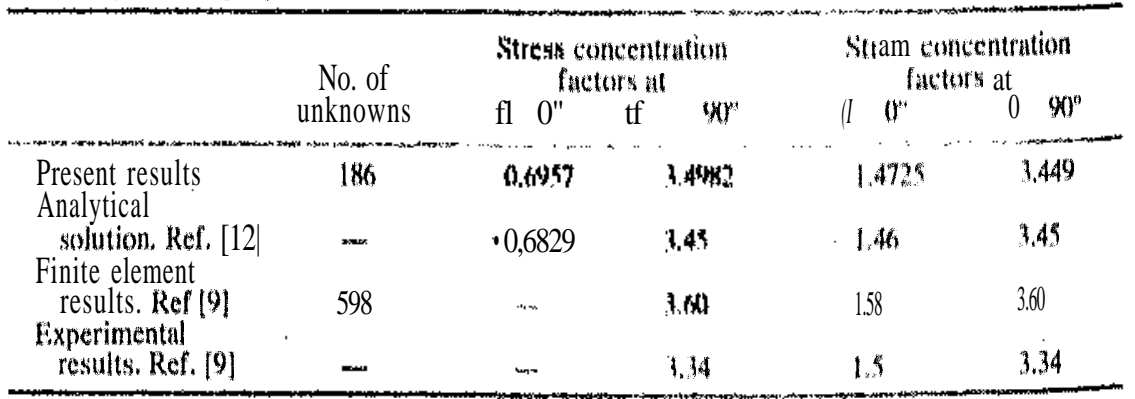




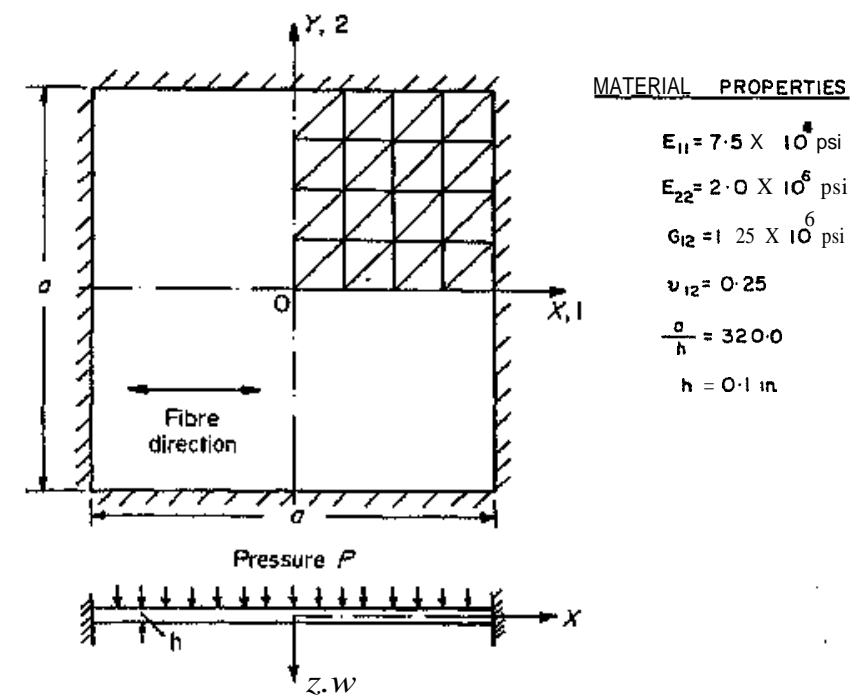

Fig. 6. Finite element grid for clamped glass-epoxy composite square plate.

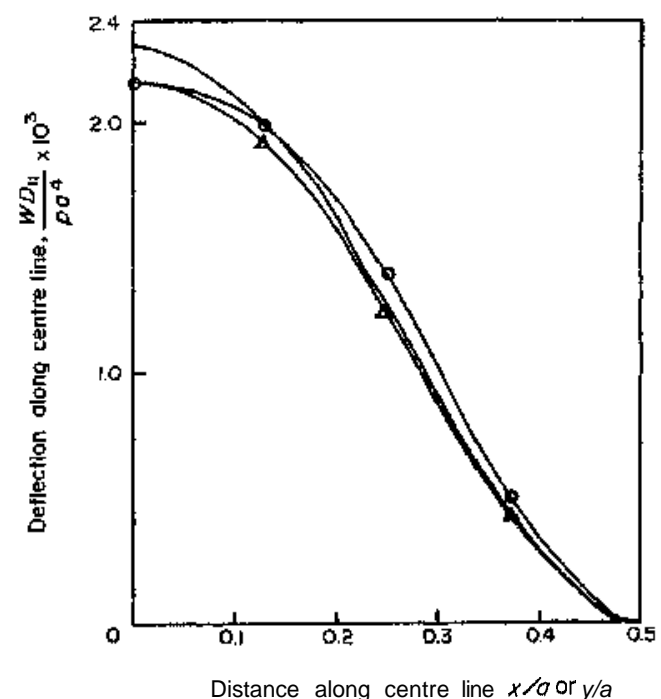

Fig. 7. Deflections of clamped glass-epoxy composite square plate. , Analytical solutions; $\mathrm{A}$, along $\mathrm{X}$-axis; 0 , along $Y$-axis.

and deflections is not possible due to inherent approximations in the available solution in Ref. [13]. (The soluion in [13] when specialized to isotropic case shows an error of about $6.3 \%$ for maximum deflection and unacceptable accuracy for maximum moments.)

An orthotropic cylindrical shell with a circular hole subjected to axial tension. The problem illustrated in Fig. 9 is analysed to evaluate the coupled membrane-bending behaviour of the cylindrical shell form of the element. Taking advantage of symmetry, only one quarter of the shell needs to be analysed. Choosing the $5 \times 5$ finite element grid illustrated in Fig. 3, 51 elements with 37 nodes (367 degrees of freedom) are used to model the quarter of the shell. The adequacy of the chosen grid to solve the present problem was checked by solving an otherwise identical isotropic shell problem. Typical results are given in Table 5 along with continuum solutions [15] for comparison to demonstrate the accuracy achieved with the rather coarse mesh used.

Predicted stress distributions for both isotropic and orthotropic shells are shown in Figs. 10 and 11 along With

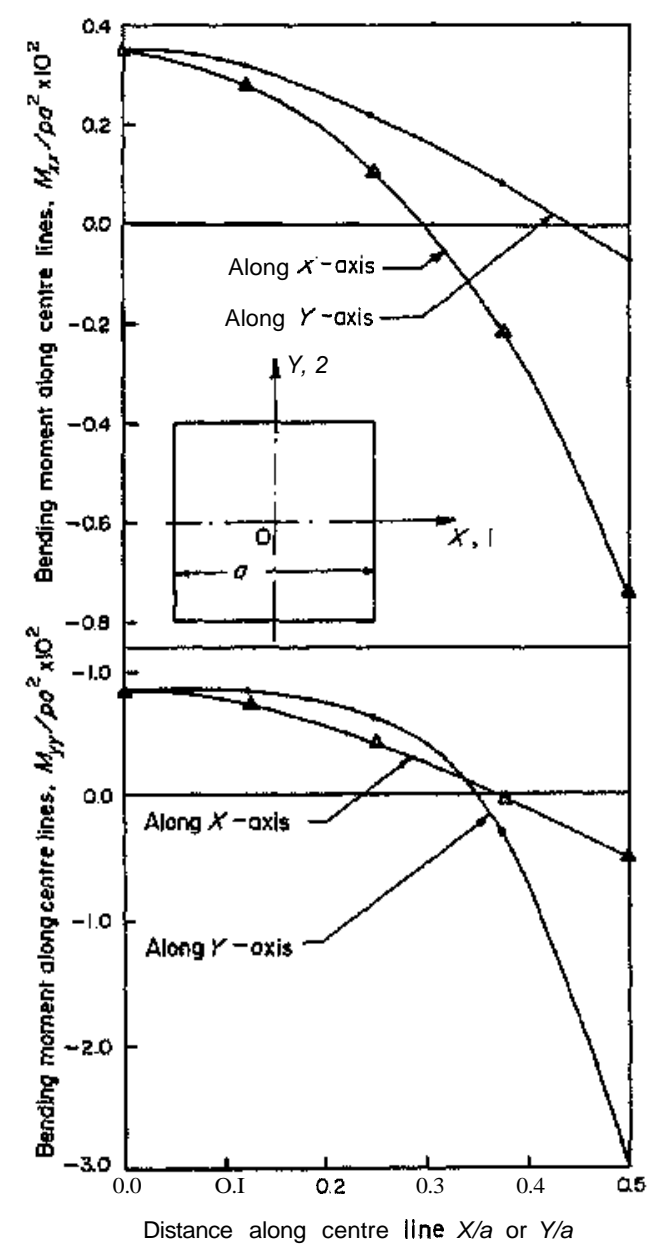

Fig. 8. Moment distributions along centre lines of clamped glassepoxy composite square plate.

the results of anisotropic elasticity solution to the problem of an infinite plate with a circular hole [11]. The predicted stresss distributions are fairly smooth and appear to have converged as well. Near the hole the coupled membrane-bending behaviour appears to be 
Table 4. Clamped glass-epoxy composite square plate subjected to uniform pressure loadcomparison of results

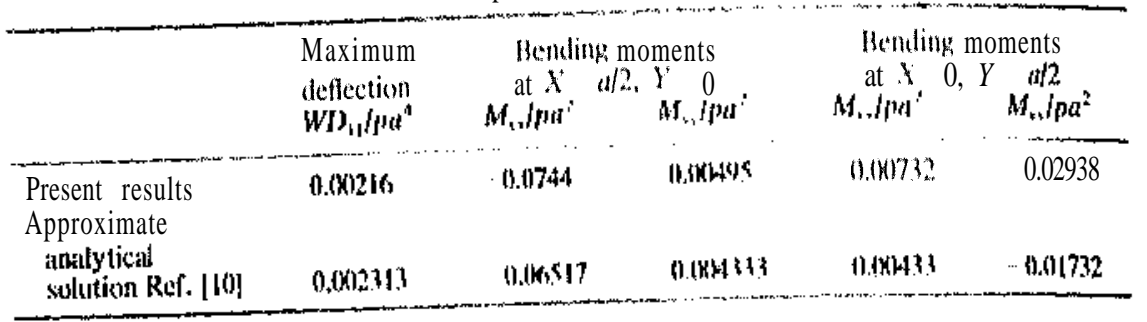

Table 5 Cylindrical shell with a circulat hole under axial tension Comparison of results

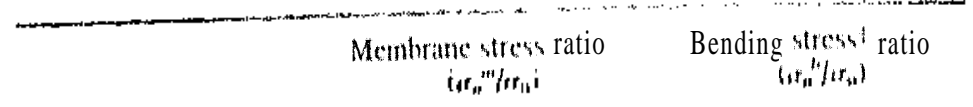

\begin{tabular}{|c|c|c|c|c|}
\hline Orthotropic, shell & int $17 \quad 16^{*+1}$ & at H & at $\|$ it & He if $4010^{\prime \prime}$ \\
\hline Present resulls & $0 ., 81,374 k$ & 49849 & 11. $3111 k ?$ & $0.4(3) 1$ \\
\hline $\begin{array}{l}\text { Orthotropic plate } \\
\text { Solution Rel, }|12|\end{array}$ & $9.481\}$ & $4, x^{4} H, 3$ & 11.11 & 0.0 \\
\hline $\begin{array}{l}\text { Isotropic shell } \\
\text { Present results }\end{array}$ & 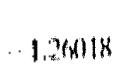 & $3.742 ?$ & 81,1499 & $0,1,3 ! !$ \\
\hline $\begin{array}{l}\text { Isotropic shell } \\
\text { results from Ref, |1।| }\end{array}$ & 1.94 & 1.5. & औ kll & $\begin{array}{ll}10.44 \\
\cdots \ldots\end{array}$ \\
\hline
\end{tabular}
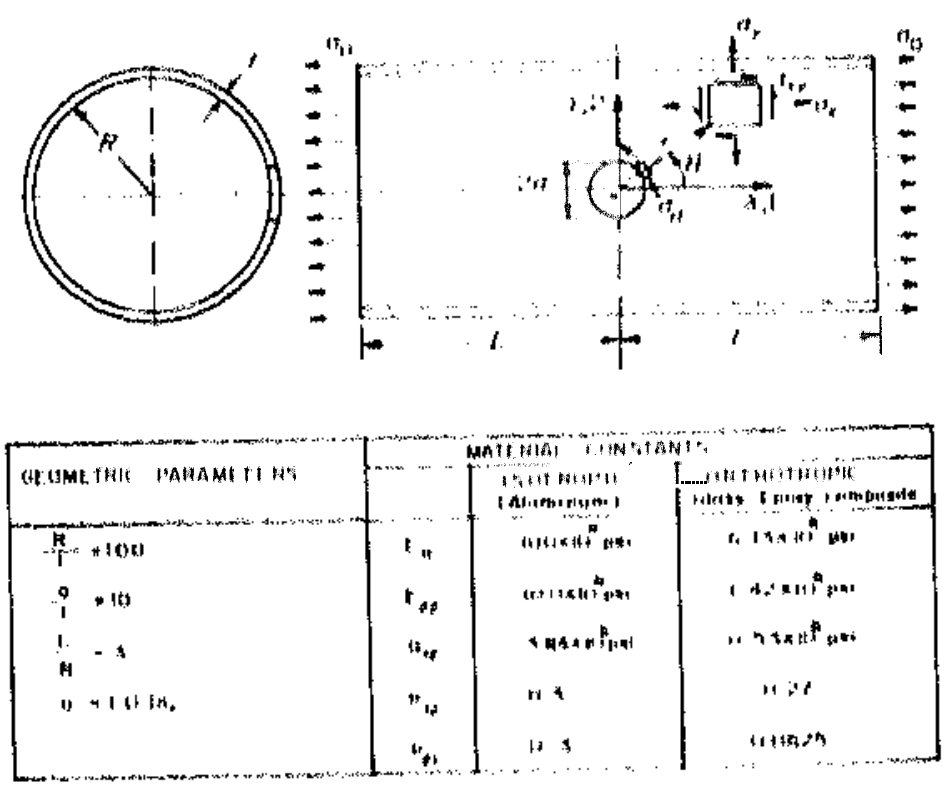

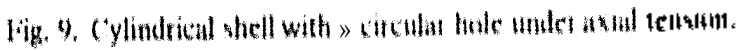

characterized adequately by the small number of elc ments used. Predieted membrane and hending stress concentration factors are presented in "rable 1 The main difficulty in assessing accuracy of these results artses from the fact that authors coud not find any other result for the orthotropic shell for comparison. Since the wo. tropic shell is a particular ease of the orthotropic shell treated here it might be expected that the same arder of accuracy holds good for both.

\section{CONCLUSIONS}

The stiffness matrix for a high precision triangular been formulated. and couled into a a posite structural

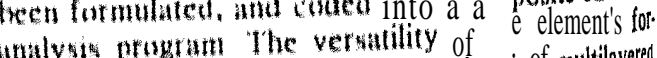
sullation cnables its use in the antaly ; of multilayered compostc plate and cylindrical shel structures taking into atcount ustual lamination parar ters. The results the we of the sub] it finite element end er stress as defoumstated gowd cotinecring accl splications with well us displacencuts in the example mondest number of elements.

the ultimate objective of any and is is as a deig toot this work implies the iterative ie of the milyt protedures in a design excretse. The resent fintedt ment formulation is ideally suited 1 c compoter ats

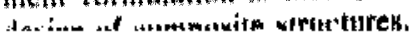




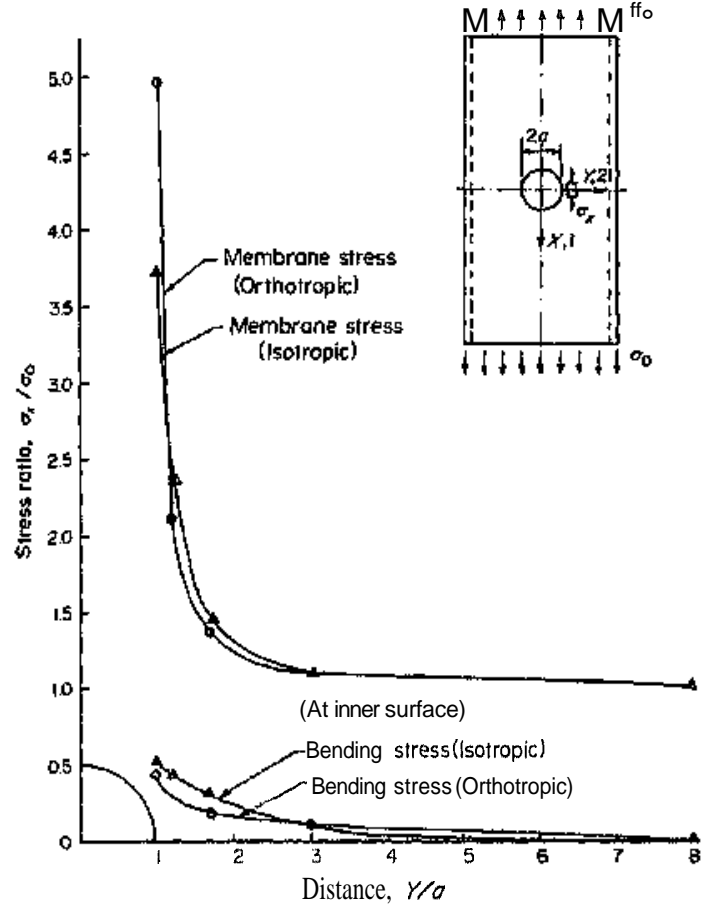

Fig. 10. Axial stress distribution along $Y$-axis.

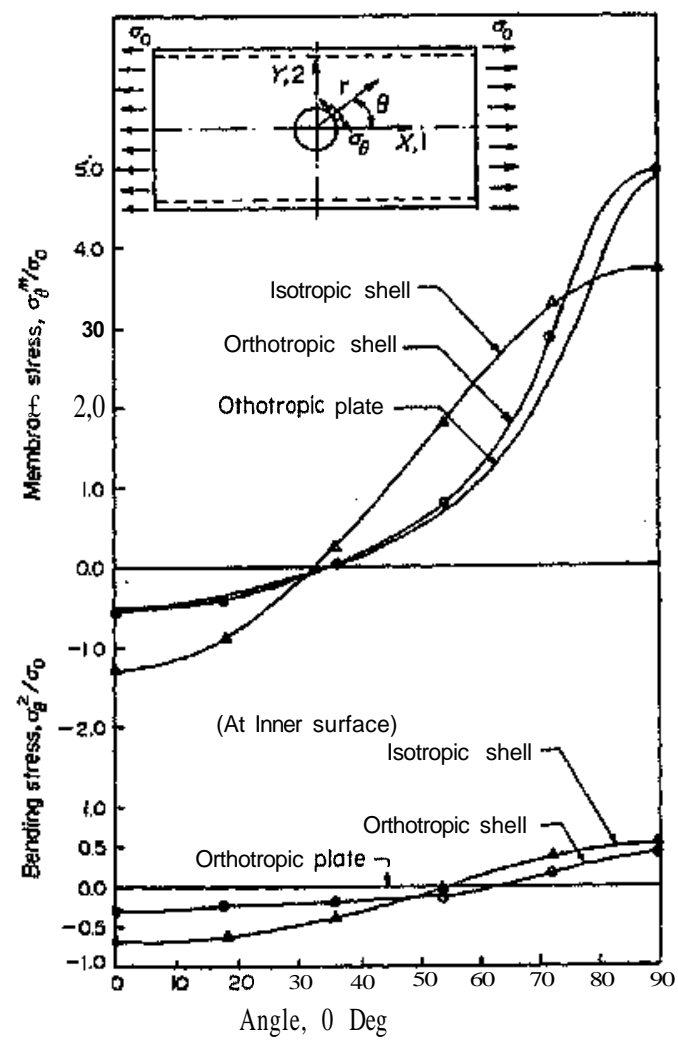

Pig. 11. Tangential stress distribution along the hole boundary.

\section{REFERENCES}

1. L. R. Calcote, The Analysis of Laminated Composite Structures. Van Nostrand, New York (1969).

2. R. H. Gallagher, Analysis of plate and shell structures. Proc. Conf. Appl. Finite Element Methods in Civil Engng, Vanderbilt University, U.S.A. pp. 155-206 (Nov. 1969).

3. R. H. Gallagher, Application of finite element analysis. In
Advances in Computational Methods in Structural Mechanics and Design. pp. 641-678 (Edited by J. T. Oden et al.) UAH Press (1972).

4. G. Dupuis, Application of Ritz method to thin elastic shell analysis. Trans ASME, J. Appl. Mech. 38, Ser. E, (4) 787 (1971).

5. G. R. Cowper, G. M. Lindberg and M. D. Olson, A shallow shell finite element of triangular shape. Int. J. Solids Struct. 6, 1133-1156(1970).

6. J. H. Argyris and D. W. Scharpf, The SHEBA family of shell elements for the matrix displacement method. Aeronautical J., 72(697), 873-883 (Oct. 1968).

7. D, J. Dawe, Higher-order triangular finite element for shell analysis. Int. J. Solids Struct. 11(10), 1097-1110(1975).

8. G. M. Lindberg and M. D. Olson, A high precision triangular cylindrical shell finite element. AIAA J. 9, 531-532 (1971)

9. G. R. Cowper, E. Kosko, G. M. Lindberg and M. D. Olson, A high precision triangular plate bending element. Aeronautical Report LR-514, National Research Council of Canada, Ottawa (Dec. 1968).

10. V. V. Novozhilov, The Theory of Thin Shells. 2nd Edn. Noordhoff, Amsterdam (1964).

11. S. G. Lekhnitskii, Theory of Elasticity of Anisotropic Elastic Body (Translated by P. Fern, Edited by J. L Brandstatter) Holden-day, San Fransisco (1963).

12. I. M. Daniel, R. E. Rowlands and J. B. Whiteside, Deformation and failure of Boron-epoxy plate with circular hole. Analyses of test methods for high modulus fibers and composites, STP 521, ASTM pp. 143-164 (1973).

13. J. E. Ashton and J. M. Whitney, Theory of laminated plates. In Progress in Material Science Series, Vol. IV, pp. 51-55. Techomic (1970).

14. A. Subramanian et al., Holographic analysis of some engineering components. NAL Technical Memorandum, TM-PR-ST201/127-76.

15. P. Van Dyke, Stresses about a circular hole in a cylindrical shell. AIAA J., 3(9), 1733-1742 (1965).

$$
\begin{aligned}
& \text { APPENDIX } \\
& \text { Stiffness Matrix [k] } \\
& k_{i j}=\tilde{L}_{11} m_{i} m_{j} F\left(m_{i}+m_{j}-2, n_{i}+n_{j}\right) \\
& \jmath \geqslant i+\bar{L}_{12}\left(m_{i} n+m_{j} n_{i}\right) F\left(m_{i}+m_{j}-1, n_{i}+n_{j}-1\right) \\
& +\tilde{C}_{13} m_{j} p_{j} F\left(m_{i}+p_{j}-2, n_{i}+q_{j}\right) \\
& +\tilde{L}_{14} m_{i} q_{j} F\left(m_{i}+p_{j}-1, n_{i}+q_{j}-1\right) \\
& +L_{15} m_{i} F\left(m_{i}+r_{j}-1, n_{i}+s_{j}\right) G_{j} \\
& +\bar{L}_{16} m_{i} r_{j}\left(r_{j}-1\right) F\left(m_{i}+r_{j}-3, n_{i}+s_{j}\right) \\
& +L_{17} m_{i} r_{j} s_{i} F\left(m_{i}+r_{j}-2, n_{i}+s_{i}-1\right) \\
& +\bar{L}_{1:} m_{i} s_{j}\left(s_{j}-1\right) F\left(m_{i}+r_{j}-1, n_{i}+s_{j}-2\right) \\
& +\bar{L}_{2 n_{i} n_{i}} F\left(m_{i}+m_{j}, n_{i}+n_{i}-2\right) \\
& +\bar{L}_{2} t_{i} p_{j} F\left(m_{i}+p_{i}-1, n_{i}+q_{j}-1\right) \\
& +\bar{L}_{24} n_{i} q_{j} F\left(m_{i}+p_{j}, n_{i}+q_{j}-2\right) \\
& +\tilde{L}_{25} n_{i} F\left(m_{i}+r_{i}, n_{i}+s_{j}-1\right) G_{j} \\
& +\bar{L}_{26} n_{i} r_{j}\left(r_{j}-1\right) F\left(m_{i}+r_{j}-2, n_{i}+s_{j}-1\right) \\
& +\tilde{L}_{27} n_{i} r_{i} s_{j} F\left(m_{i}+r_{j}-1, n_{i}+s_{i}-2\right) \\
& +\bar{L}_{28} n_{j} s_{j}\left(s_{j}-1\right) F\left(m_{i}+\text { fy } n_{i}+s_{j}-3\right) \\
& +\tilde{L}_{33} p_{i} p_{j} F\left(p_{i}+p_{j}-2, q_{i}+q_{j}\right) \\
& +\tilde{L}_{34}\left(p_{i} q_{j}+p_{j} q\right) F\left(p_{i}+p_{j}-1, q,+q_{j}-1\right) \\
& +\tilde{L}_{3 s} p_{i} F\left(p_{i}+r_{i}-1, q_{i}+s_{j}\right) G_{j} \\
& +L_{3 i} p_{i} r_{j}\left(r_{j}-1\right) F\left(p_{i}+r_{j}-3, q_{i}+s_{j}\right) \\
& +L_{3 \uparrow} p_{i} s_{p} F\left(p_{i}+r_{j}-2, q_{i}+s_{i}-1\right) \\
& +\tilde{L}_{38} p_{j} s_{j} s_{i-1} F\left(p_{i}+r_{j}-1, q,+s_{j}-2\right) \\
& +\tilde{L}_{4} q_{A} q_{l} F\left(p_{1}+p_{\mathrm{j}} q_{1}+q_{1}-2\right) \\
& +\tilde{L}_{4 s} q_{i} F\left(p_{i}+r_{j}, q_{i}+s_{j-1}\right) G_{i} \\
& +\tilde{L}_{46} q_{i} r_{j}\left(r_{i-1}\right) F\left(p_{i}+r_{j}-2, q_{i}+s_{j}-1\right) \\
& +\bar{L}_{47} q_{i} r_{i} s_{t} F\left(p_{t}+r_{j}-1, q_{i}+s_{j}-2\right)
\end{aligned}
$$




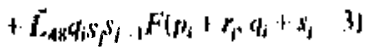

$+\bar{L}_{58} F\left(r_{i}+r_{j}, s_{i}+s_{1}\right) G_{i}\left(i_{1}\right.$

$+\bar{L}_{s b} r_{j}\left(r_{1} \cdots 1\right) f\left(r_{i}+r_{i} \cdots 2, s_{i}+s_{i}\right)\left(i_{i}\right.$

$+L_{s} r_{j} s_{j} f\left(r_{i}+r_{i} \cdot\left\{, s_{i}\right) s_{i} \cdot 1\right]\left(i_{i}\right.$

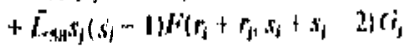

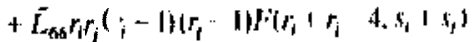

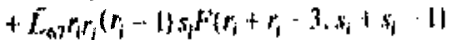

$\left.+I_{r i n}\left|r_{i} s_{j}\left(r_{i}-1\right)\left(s_{j} \cdots 1\right)+r_{r} s_{t}\right| r_{3} \quad \mid\right)\left(s_{i} \cdot \mid\right) \mid$

$\times F\left(r_{i}+r_{i}-2 . n_{i}+s_{j}-2\right)$

$+i_{n} r_{i} r_{i} r_{i} F_{j} F\left(r_{i}+r_{j} \cdots 2 . s_{1}+s_{j} \cdot 3\right)$

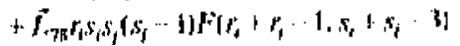

If b.

wiklt:

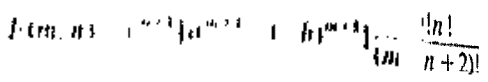

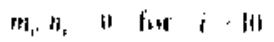

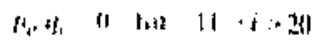

$t_{t} x_{1} \|$ fat $i$ - .

if 18 fin 1.3.

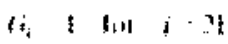

